\title{
INVESTIGACIONES
}

\section{Análisis crítico del discurso de las competencias en la formación inicial docente en Chile}

\author{
Critical Discourse Analysis of Competency-based Curriculum \\ in Initial Teacher Training in Chile
}

\author{
Análise crítica do discurso de competências na formação \\ inicial de professores no Chile
}

\author{
Olga Espinoza Aros. ${ }^{a}$ \\ ${ }^{\text {a }}$ Facultad de Educación, Pontificia Universidad Católica de Chile. Fono: 56-2-223545350. \\ Correo electrónico: omespino@uc.cl
}

\begin{abstract}
RESUMEN
En el contexto de la globalización neoliberal, la educación superior se ha visto impactada por procesos diversos y complejos; en este escenario, el currículum por competencias se ha posicionado como la respuesta preponderante a los actuales desafíos educativos. En Chile, la formación inicial docente es mayoritariamente de carácter universitario, por tanto, también se ha visto afectada por tales transformaciones y demandas. En este artículo se presenta un análisis que problematiza las condiciones de producción del discurso de las competencias en instituciones de formación inicial docente, para ello, se realiza un análisis del discurso de académicos que participaron en comités curriculares, elaborando currículos de formación docente basados en competencias. Se parte de la comprensión del currículum como una práctica discursiva y se aborda desde la perspectiva teóricometodológica del Análisis Crítico del Discurso, de orientación foucaultiana.
\end{abstract}

Palabras clave: currículum por competencias, análisis crítico del discurso, formación inicial docente.

\begin{abstract}
In the context of neoliberal globalization, higher education has been affected by many complex processes. In this context, the Competency-based Curriculum has emerged as a predominant response to current educational challenges. In Chile, initial teacher training programs in universities have been affected by such changes and demands. This paper presents an analysis whose objective is to problematize the conditions of production of discourse of competencies in teacher training programs in universities. To fulfill this purpose, it is presented an analysis of interviews to academics who participated in curriculum committees, and who also developed teacher training competency-based curricula. In this paper curriculum is understood as a discursive practice and a Foucauldian critical discourse analysis is used.
\end{abstract}

Key words: competency-based curriculum, critical discourse analysis, initial teacher training programs.

\section{RESUMO}

No contexto da globalização neoliberal, o ensino superior foi impactado por processos variados e complexos; neste cenário, o currículo por competências tem ficado como a principal resposta aos atuais desafios educativos. No Chile, a formação inicial de professores é principalmente de caráter acadêmico e, portanto, também tem sido afetada por tais transformações e demandas. Apresenta-se uma análise que problematiza as condições de produção do discurso das competências nas instituições de formação inicial de professores; para isso, faz-se uma análise do discurso de acadêmicos que participaram em comitês curriculares desenvolvendo currículos de formação de professores baseados em competências. Inicia-se na compreensão do currículo como uma prática discursiva e é abordado a partir da perspectiva teorico-metodologica foucaultiana.

Palavras chave: currículo competência, análise crítica do discurso, formação inicial de professores. 
[el discurso es] un bien -finito, limitado, deseable, útilque tiene sus reglas de aparición, pero también sus condiciones de apropiación y de empleo; un bien que plantea, por consiguiente, desde su existencia (y no simplemente en sus "aplicaciones prácticas") la cuestión del poder; un bien que es, por naturaleza, el objeto de una lucha, y de una lucha política (Foucault, 2002: 204).

\section{INTRODUCCIÓN}

Toda aproximación al campo curricular implica definir una postura teórica respecto del concepto de currículum, ya sea concibiéndolo como racionalidad (Grundy, 1994), como ideología (Schiro, 2008), como práctica discursiva (Cherryholmes, 1999; Pinar et al., 2008; Popkewitz y Brennan, 2000; Silva, 2001a, 2001b, 1998), entre otras posibilidades. En este artículo se comprende el currículum como una práctica discursiva, de modo que éste se entiende como "una práctica de significación, como un texto, como una trama de significados; [por tanto] el currículum puede ser analizado como un discurso" (Silva, 1998: 68). Desde esta perspectiva, analizar las relaciones de conocimiento y poder presentes en los currículos es un ejercicio de deconstrucción sobre la voluntad de verdad que los mueve, para remontarse a la pregunta de por qué ese conocimiento se volvió verdadero: "Siguiendo a Foucault, una perspectiva postestructuralista sobre el currículum desconfía de las nociones filosóficas de 'verdad' [....] abandona de modo radical el énfasis en 'la verdad' para destacar el proceso por el cual algo se considera la verdad” (Silva, 2001a:151).

La construcción curricular siempre es un proceso complejo, que involucra dimensiones políticas y técnicas; implica toma de decisiones, las cuales poseen un carácter ideológico, ya que representan las visiones de sujeto y de conocimiento que una propuesta educativa pretende desarrollar (Gvirtz y Palamidessi, 2005; Moreno, 2008; Schiro, 2008; Silva, 2001a, 2001b, 1998). Si bien todo proceso de construcción curricular puede generar un interés investigativo, la introducción del currículum por competencias a la formación de carácter universitario es especialmente relevante, ya que se trata de un modelo curricular que tiene sus antecedentes en la gestión empresarial y en la formación técnica y laboral, y no en el ámbito académico universitario. Por tanto, desde la comprensión del currículum como una práctica discursiva, surge el interés por comprender las condiciones de producción de este discurso en el ámbito universitario, o sea, cómo se introduce, cómo se legitima y cómo se concreta en las decisiones curriculares, en este caso, del currículum de la formación inicial docente.

En este artículo se presenta parte de una investigación sobre el discurso de las competencias en el contexto de la educación superior universitaria, cuya propuesta teóricometodológica es una aproximación desde el Análisis Crítico del Discurso de orientación foucaultiana (Jager, 2009, 2008, 2003). La utilización de la conceptualización foucaultiana posibilitó problematizar las condiciones de producción del discurso de las competencias en la educación superior universitaria en general, y en la formación inicial docente en particular. En este trabajo se ha optado por la perspectiva foucaultiana debido al aporte de ésta al planteamiento de interrogantes frente a un tema que preponderantemente se ha descrito en términos operacionales y se ha profundizado poco en su análisis crítico, así que, como indica Veiga-Neto (1997), se ha tomado a Foucault "como el inspirador de una 
teoría analítica [...] no por las respuestas que él es capaz de darnos -y que no son ni pocas ni simples-, sino tomarlo principalmente, por el tipo de preguntas que él hace nacer en nosotros" (Cit. en Rifà Valls, 2003: 45).

\subsection{LA EDUCACIÓN SUPERIOR UNIVERSITARIA EN EL CONTEXTO DE LA GLOBALIZACIÓN NEOLIBERAL}

La globalización es un fenómeno complejo tanto por la heterogeneidad de los procesos y mecanismos implicados en su desarrollo como por los efectos que produce en distintos ámbitos, no sólo a nivel económico, sino también en lo político, social y cultural. En educación, si bien no todos los cambios de los sistemas educativos a nivel mundial en las últimas décadas se pueden atribuir al efecto de la globalización, existen antecedentes relevantes para afirmar que ha generado importantes transformaciones, tanto en las políticas como en los procesos derivados de éstas (Bonal, Tarabini-Castellani y Verger, 2007; Dale, 2007; Green, 2007; Santos, 2005). López Segrera (2003), refiriéndose al contexto latinoamericano, afirma que la globalización, en su dimensión neoliberal, ha impactado profundamente a los sistemas educativos latinoamericanos, desde fines del siglo veinte hasta la actualidad. Dicha aseveración es válida para la educación a escala mundial, ya que los efectos de la globalización en la educación son de un amplio espectro, atañen tanto a las decisiones macro políticas como a aspectos más específicos, tales como las definiciones curriculares, aspecto que se abordará en el presente artículo.

Otra característica relevante respecto de la complejidad de los procesos y de los efectos de la globalización en el ámbito educativo, es que ésta no conlleva a una anulación del rol del Estado, sino que más bien, a través de diversas prácticas, se promueven agendas similares de política educativa, las que contemplan desafíos o problemáticas comunes (Dale, 2007), lo cual redunda en que se promueva también la generación de mecanismos de respuesta comunes. Un ejemplo de estos mecanismos son las respuestas transnacionales a las diversas demandas actuales de la educación superior, como los acuerdos del Espacio Europeo de Educación Superior ${ }^{1}$ o las acciones que contempla el Proyecto Tuning (iniciado en Europa y posteriormente extendido a América Latina, África, Estados Unidos y Rusia).

La educación superior es uno de los segmentos más afectados por la globalización neoliberal (Ibarra, 2003; López Segrera, 2007, 2003; Slaugther \& Rhoades, 2004). Al analizar las dinámicas de desarrollo y cambio de la educación superior desde las últimas décadas del siglo pasado, se pueden identificar seis procesos clave que permiten comprender las condiciones de producción del discurso de las competencias en este nivel educativo, éstos son: la masificación de la matrícula, los procesos de mercantilización de la educación superior, la vinculación más estrecha de los programas de formación con las necesidades del mercado laboral, los procesos de internacionalización, la instauración de sistemas de aseguramiento de la calidad, y la promoción de 'patrones de respuesta' a los desafíos educativos globales.

Estos procesos se han generado interrelacionadamente y actúan como fuerzas que presionan a los sistemas de educación superior, poniendo de relieve problemáticas que tienen directa relación con definiciones curriculares. Tanto para los requerimientos producidos por la expansión de la educación superior como para los procesos de aseguramiento de

En adelante E.E.E.S. 
la calidad, el modelo curricular por competencias se ha posicionado en el discurso como el patrón de respuesta que permitiría resolver las exigencias de pertinencia y efectividad de los procesos formativos. Por tanto, resulta altamente relevante atender a la vinculación entre los procesos de cambio de la educación superior en términos globales y su impacto en las definiciones curriculares; como indica Silva, el alcance de las políticas curriculares son de orden político, social y epistemológico:

\begin{abstract}
las políticas curriculares, constituyen al menos, como texto, como discurso, un importante elemento simbólico del proyecto social de los grupos de poder [...] Las políticas curriculares tienen también otros efectos. Autorizan a ciertos grupos de especialistas, al mismo tiempo que desautorizan a otros. Fabrican los objetos "epistemológicos" de los que hablan, a través de un léxico propio, con una jerga que no debe verse como una moda, sino como un mecanismo muy eficiente de institucionalización y constitución de lo "real", que supuestamente le sirve de referente (1998: 62).
\end{abstract}

La preponderancia que ha adquirido el currículum por competencias en la educación en general, y en la educación superior en particular, ha impuesto un nuevo lenguaje en el quehacer curricular universitario, el cual no sólo es una nueva forma de definir los propósitos formativos, sino una conceptualización respecto del conocimiento, de la formación universitaria y de los sujetos en formación.

\title{
2. METODOLOGÍA
}

La propuesta teórico-metodológica que se generó en esta investigación se basa en los planteamientos sobre discurso y sobre análisis de discurso que desarrolla Michel Foucault en La arqueología del saber ([1969] 2002) y El orden del discurso ([1970] 2005). La primera etapa de la investigación, contempló el estudio en profundidad de estas obras, para luego generar el levantamiento de lo que se denominó "Cajas de herramientas foucaultianas para el análisis de discurso". Éstas corresponden a una selección y organización de conceptos foucaultianos sobre discurso y análisis de discurso, los cuales se utilizaron como categorías de análisis. Con estas herramientas se desarrollaron los procedimientos metodológicos, los que se agruparon en tres ámbitos: la generación del corpus discursivo, la puesta en relación del corpus discursivo con las categorías de la descripción arqueológica y la construcción del texto de descripción arqueológica.

La descripción arqueológica es la propuesta de análisis de discurso que plantea Foucault $(2005,2002)$ en las obras anteriormente mencionadas; esta perspectiva analítica no tiene como propósito dar cuenta del origen del discurso o realizar un análisis hermenéutico para desentrañar el sentido oculto de éste, sino que se trata de describir regularidades discursivas que permitan dar cuenta de las condiciones de producción. Por otra parte, también contempla la descripción de contradicciones, cuyo propósito no es reducirlas o proponer soluciones para superarlas, sino mostrar las discontinuidades y quiebres de las formaciones discursivas, por tanto, la complejidad y densidad de su configuración.

El corpus discursivo de la investigación está constituido por: textos referidos a procesos de la educación superior universitaria (Declaraciones del E.E.E.S e Informes del Proyecto Tuning europeo y latinoamericano); textos normativos de la formación inicial docente, el 
texto generado en 17 entrevistas a académicos que participaron en comités curriculares de proyectos de renovación curricular, y los perfiles de egreso construidos en el marco de dichos proyectos.

\section{RESULTADOS}

En este artículo se presenta sólo una parte del análisis realizado, el cual se centra en la descripción de las condiciones de producción del discurso de las competencias en la formación inicial docente. Para ello se abordará la entrada al orden del discurso, es decir, cómo se introduce en el quehacer académico, cuáles son las diferentes perspectivas de apropiación de éste por parte de los sujetos y, finalmente, las contradicciones del discurso.

\subsection{LA ENTRADA AL ORDEN DEL DISCURSO DE LAS COMPETENCIAS A TRAVÉS DE LA CONSTRUCCIÓN CURRICULAR}

Si bien se puede afirmar que desde el Proyecto FFID $^{2}$ en adelante se comienza a hablar de competencias en la formación inicial docente, el discurso de las competencias se introduce de modo decisivo y explícito en el quehacer curricular de las instituciones de formación docente a través de los proyectos de renovación curricular financiados por el Programa de Mejoramiento de la Calidad de la Educación Superior (MECESUP).

Desde la perspectiva de la descripción arqueológica foucaultiana, los llamados a concurso de renovación curricular pueden ser entendidos como la superficie de emergencia del discurso, porque a través de éstos se integra al quehacer académico universitario. En los términos de referencia de estos proyectos se identifica la presencia de uno de los mecanismos de control discursivo, que Foucault denomina comentario, el cual se refiere a la presencia de unos discursos en otros. Así pues, se puede observar que los términos de referencia replican los enunciados de la convergencia, propios del discurso curricular del E.E.E.S y del Proyecto Tuning, ya que tanto en sus lineamientos generales como en los requisitos de ejecución, se apela a los mismos argumentos de armonización y convergencia curricular, lo cual se lograría mediante el desarrollo de proyectos de carácter interinstitucional a través de consorcios de universidades, en una lógica de patrones de respuesta comunes a desafíos comunes.

Por tanto, serán prácticas institucionales las que introducen el discurso de las competencias en el ámbito universitario al decidir su participación en estos proyectos. Las instituciones universitarias entran en el orden del discurso de las competencias, adscribiendo a las políticas curriculares que tienen como propósito sintonizar con las tendencias internacionales de educación superior, orientadas tanto a la convergencia de las propuestas curriculares, como a la responsabilización de las instituciones por los procesos formativos. Además, la motivación institucional también se relaciona con la posibilidad de acceder a los recursos económicos que estos proyectos implican, y también a posicionarse o a mantener un posicionamiento ante otras instituciones de educación superior.

El Proyecto FFID (Fortalecimiento de la Formación Inicial Docente) fue impulsado por el Ministerio de Educación y se desarrolló entre 1998 y 2002; en él se generó el primer documento de estándares de desempeño para egresados de la formación inicial docente. 
Cuando los académicos fueron convocados a participar en los comités curriculares, se encontraron ante un tema complejo; independiente de la opinión que tuviesen respecto del currículum por competencias, la mayoría manifestó que la tarea de generar un diseño curricular de este tipo significó enfrentarse a algo nuevo y desconocido, para lo cual no se sentían preparados. Por tanto, hubo una etapa inicial de cierta confusión e inseguridad al sentir que no poseían los conocimientos necesarios para el diseño de las propuestas curriculares; y durante el desarrollo de los proyectos, algunos percibían que incluso a nivel institucional tampoco existía suficiente claridad sobre el tema.

Para otros, la complejidad se relacionaba con el reconocimiento de los antecedentes conceptuales del discurso de las competencias, el cual se vincula con una perspectiva curricular técnica y de eficiencia social, lo cual les parecía disonante con el carácter académico de la formación universitaria. Estas problemáticas dan cuenta de cómo operan los discursos: éstos se reproducen y circulan mediante prácticas institucionales, ya que son éstas las que deciden participar o se sienten presionadas a asumir este discurso curricular, sin embargo, esta nueva práctica curricular tenía escasa relación con los contextos, con las actividades académicas, y con los significados atribuidos por los sujetos, quienes debieron asumir un nuevo rol: el de diseñadores curriculares bajo este modelo.

Las dificultades a las que se enfrentaron fueron de tipo conceptual y técnico. En el plano conceptual, la diversidad de definiciones y enfoques respecto de las competencias, provocó en algunos equipos la necesidad de realizar una inmersión profunda en el modelo curricular, lo cual fue considerado como una oportunidad para acercarse a un tema desconocido; algunos equipos destacaron esta experiencia como interesante y relevante para la comprensión de los actuales desafíos de la formación profesional universitaria. Sin embargo, otros plantearon una visión crítica al respecto, ya que consideraron que no hubo suficiente discusión conceptual previa, lo que debilitó la fundamentación de las perspectivas teóricas asumidas y demoró los procesos.

La dificultad para definir referentes y enfoques se relaciona con la diversidad de definiciones y clasificaciones que suelen presentarse en la literatura referida al currículum por competencias. Desde la perspectiva del análisis del discurso, esta diversidad de campos de enunciados se relaciona con la discontinuidad de los discursos, ya que éstos no tienen un desarrollo lineal, sino que las formaciones discursivas se relacionan complejamente, incluso de modo incompatible y contradictorio; es a través de su puesta en relación, mediante reglas de producción, como circulan a través de prácticas institucionales que legitiman su uso y apropiación. El discurso de las competencias que se instala en la formación profesional universitaria posee la remanencia del discurso de las competencias del ámbito laboral y de la formación técnica, es decir, conserva enunciados como el análisis de tareas y cierta orientación conductista respecto del aprendizaje. Sin embargo, conjuntamente, se presentan nuevas conceptualizaciones de las competencias, vinculadas al ámbito escolar y académico, en las que la orientación respecto del aprendizaje es más cercana al constructivismo. Debido a la escasa discusión teórica con que generalmente se presentan las definiciones y los procedimientos de este modelo curricular, estas distinciones conceptuales se tornan difusas y confusas.

Los equipos académicos enfrentaron la necesidad de comprender el modelo curricular basado en competencias, y además asumir los requerimientos técnicos de su diseño; la etapa que dedicaron a la comprensión y apropiación del discurso de las competencias no había sido considerada en los términos de referencia, lo cual redundó en que se cuestionara 
el tiempo destinado a este proceso inicial porque retardó la ejecución proyectada. En términos discursivos, se aprecia que desde las instancias institucionales, se supuso que la entrada al orden del discurso era un proceso de menor complejidad.

Finalmente, cabe indicar que los Proyectos Tuning europeo y latinoamericano, cumplen un rol determinante en este proceso de entrada al orden discursivo; éstos operan como una instancia de delimitación (rol institucional en la definición del objeto del discurso), y rejilla de especificación (sistemas de clasificación y especificación de los objetos del discurso), ya que los documentos y lineamientos de los Proyectos Tuning europeo y latinoamericano, serán importantes referentes conceptuales que influirán tanto en la definición del concepto de competencias que se adoptó en los proyectos de renovación curricular, como en la orientación metodológica para la definición de las competencias.

\subsection{PERSPECTIVAS DE APROPIACIÓN DEL DISCURSO}

En el proceso de entrada al orden del discurso, los equipos académicos manifiestan distintas perspectivas respecto del discurso de las competencias: algunos plantean una valoración positiva, concibiéndolo como una oportunidad de cambio efectivo de la formación docente; otros plantean una visión cuestionadora tanto del modelo como del proceso; y, finalmente, hay quienes se posicionan desde una perspectiva más bien pragmática.

En general, los académicos entrevistados manifestaron una opinión crítica respecto de la calidad de la formación inicial docente; las principales deficiencias que identificaron son: descontextualización respecto de las necesidades de las instituciones escolares, predominio de la enseñanza centrada en los contenidos y no orientada a la profesionalización, falta de reflexión y desvinculación de las instituciones de formación docente respecto de los requerimientos del sistema, específicamente de las definiciones que ha planteado la política educativa. En este escenario el currículum por competencias se concibe como una propuesta necesaria y pertinente; el argumento de la pertinencia se sustenta en que éste implicaría no sólo una modificación de las estructuras curriculares, sino también un cambio en la concepción de la formación, el cual se asocia a la posibilidad de explicitar con mayor exactitud los propósitos formativos, a otorgar mayor relevancia al saber-hacer, a la innovación en las metodologías de enseñanza y de evaluación, entre otros aspectos.

Desde esta perspectiva de apropiación del discurso, los cuestionamientos al currículum por competencias son considerados 'críticas ideologizadas', las cuales no comprenderían que ésta es una tendencia educativa contemporánea, respecto de la cual habría consenso, por cuanto permitiría responder efectivamente a las necesidades actuales de la educación superior en general y de la formación docente en particular. Aquí se evidencia un posicionamiento del discurso de las competencias, como un discurso verdadero, desideologizado y neutro.

Para aquellos que expresan una visión cuestionadora, el discurso de las competencias es una moda, una tendencia de carácter transitorio, como las diversas corrientes pedagógicas que se han sucedido históricamente. Lo consideran como una 'nueva forma de hablar en educación', la cual es descrita como un lenguaje vacío, un cliché, un lenguaje tautológico, que se usa como un eslogan, sólo como una declaración de intenciones que tendría escasas posibilidades reales de concreción en las prácticas educativas de las instituciones. Por otra parte, algunos indican que más que una moda, es un discurso intencionado desde las políticas públicas, lo que provoca que se 'hable' de una determinada manera. Por tanto, respecto de las condiciones de producción, se identifica que es un discurso que se ha 
instalado con mucha efectividad en la educación superior, tanto por la fuerza performativa con que se posiciona, como por las pocas instancias de reflexión crítica, ya que autoridades universitarias y académicos lo adoptarían porque es una tendencia internacional, de carácter inevitable, pero sin un análisis profundo de sus alcances y limitaciones.

Otra de las tensiones identificadas es el reconocimiento del origen del modelo de competencias y sus implicancias para la educación superior universitaria, ya que éste se orienta preferentemente hacia la formación profesional, reduciendo -en su campo de elecciones teóricas- los enunciados referidos al proyecto social, político y cultural de las instituciones universitarias, enfatizando aquellos enunciados que relacionan los fines de la educación con las demandas de la economía del conocimiento y los requerimientos del mercado laboral.

Finalmente, la tercera perspectiva de apropiación del discurso se plantea como una adhesión pragmática, ya que si bien comprenden sus condiciones de producción, asumen el modelo curricular, adaptándose o planteando críticas moderadas. Esta perspectiva está asociada a una opción estratégica, la cual no sólo tiene que ver con la obtención de recursos, sino también con la necesidad pragmática de adscribir a un discurso hegemónico, que es el que orienta actualmente las políticas de construcción curricular a nivel mundial.

\subsection{DESCRIPCIÓN DE CONTRADICCIONES DEL DISCURSO}

\subsubsection{El discurso de las competencias como patrón de respuesta para la formación inicial docente}

En el proceso de construcción curricular, el discurso de las competencias se posiciona como un patrón de respuesta puesto que se argumenta que representa la posibilidad de un cambio importante en la formación inicial docente, ya que permitiría explicitar con mayor claridad las intenciones formativas, además favorecería la innovación en la enseñanza y la superación de modelos de docencia excesivamente academicistas. Por otra parte, también se argumenta que el currículum basado en competencias favorece la integración curricular, posibilita la evaluación de los desempeños, y también el desarrollo de competencias profesionales en contextos reales. Sin embargo, si se analiza con detención cada uno de estos argumentos, se puede identificar que sólo el referido al desarrollo de desempeños profesionales en contextos reales, es propio del discurso de las competencias porque, según lo definido en este modelo curricular, las competencias se desarrollan y se ponen en práctica en contextos específicos.

La explicitación de intenciones formativas, la renovación de las concepciones sobre evaluación y métodos de enseñanza, y la integración curricular son características de cambios curriculares que no se resuelven únicamente mediante un modelo de competencias; cualquier modelo curricular permitiría la modificación o renovación de dichos elementos. Todo currículum implica una definición de intenciones formativas, por otra parte, la integración curricular es un criterio de diseño curricular (Schiro, 2008), válido para cualquier tipo de modelo. Los argumentos sobre los cuales se plantea su aporte respecto de la renovación de las prácticas de evaluación y de la innovación en las metodologías de enseñanza, tienen sus antecedentes conceptuales más bien en los modelos curriculares denominados 'centrados en el estudiante', los que no se construyen necesariamente desde una perspectiva técnica del currículum o de eficiencia social.

De lo anterior se desprende que si bien a través del discurso de las competencias se ha instalado la discusión curricular y pedagógica en la educación superior universitaria, 
este discurso ha monopolizado las comprensiones respecto de la generación de propuestas formativas, sobredimensionando sus características y posibilidades, e instalándose como único patrón de respuesta a los desafíos de la educación actual.

\subsubsection{Sistemas de exclusión en la explicitación de intenciones formativas institucionales}

Analizar las condiciones de producción del discurso implica atender a la puesta en relación de enunciados y también a las prácticas institucionales como mecanismos de regulación discursiva, es decir, cuáles son los mecanismos de inclusión y exclusión que operan en el discurso. En el discurso de las competencias se aprecia que se favorece la explicitación de los aspectos más instrumentales de los propósitos formativos, y no se relevan -e incluso se omiten- las dimensiones más complejas y problemáticas de éstos.

En relación a los lineamientos del diseño curricular por competencias, éstos contemplan procesos de análisis detallado de las tareas que conforman un desempeño y la desagregación de competencias en áreas de competencia, sub competencias, etc. Según lo analizado en esta investigación, el desarrollo del procedimiento de construcción curricular por competencias, se concentró en un trabajo excesivamente técnico, una labor 'orientada a la tarea', consistente en definir listas de competencias, llenar matrices, hacer rúbricas; proceso en el que las discusiones respecto del sentido y los propósitos de los cambios curriculares propuestos quedaron en un segundo plano.

Por tanto, estos procesos evidencian una contradicción del discurso, ya que en múltiples enunciados se plantea que el discurso de las competencias permitiría explicitar mejor las intenciones formativas de un currículo de formación profesional. Sin embargo, en los proyectos estudiados esta explicitación se focalizó en la especificación del 'saber hacer', o sea, la dimensión instruccional del ejercicio docente, pero la reflexión referida a aspectos más complejos, como la concepción de sujeto docente y la coherencia de esta concepción con la visión institucional, fue atendida en menor medida.

\subsubsection{La limitación de la disposición causal de los enunciados: Las dificultades e imposibilidades de la implementación}

En el discurso de las competencias, predominantemente, se establece una disposición causal de los enunciados, la cual se evidencia en que se atribuye al modelo curricular por competencias características que garantizarían no sólo el cambio sino también la mejora de la educación superior en general, y específicamente en la formación inicial docente. La tercera contradicción identificada tiene relación con esto, ya que en contraposición a las múltiples posibilidades atribuidas al modelo, cuando los académicos se refirieron a la implementación de las propuestas curriculares, realizaron juicios en los que identificaron importantes trabas para el éxito real de la implementación.

Las principales dificultades las atribuyeron a las lógicas institucionales que impiden el desarrollo de los cambios, la implicancia de recursos materiales y económicos, y también a dificultades en los equipos académicos. Plantearon que el proceso de diseño puede resultar más fácil si se le compara con la implementación, ya que si bien éste tenía unas directrices específicas respecto del modelo curricular a utilizar, la verdadera problemática emerge al querer concretar las intenciones declaradas. Del mismo modo, expresaron aprehensión en relación a la definición del tipo de profesional que se desea formar y que explicitaron en los 
perfiles de egreso, ya que existiría el riesgo de que éstos sean sólo una expresión de buenas intenciones, 'palabras no más', debido a que persisten muchas interrogantes respecto a su concreción efectiva en la práctica.

En términos administrativos, el discurso de las competencias entra en contradicción con las lógicas de acción y decisión institucional universitaria, que son complejas y con particularidades diversas. Los procedimientos para los cambios curriculares suelen considerar una serie de etapas administrativas que no siempre corresponden con los tiempos diseñados y comprometidos en los proyectos. El currículum por competencia también supone modificaciones a la docencia, lo cual entra en conflicto con la visión de cátedra y docencia directa propia de la universidad. Por ejemplo, el concepto de módulo que promueve el currículum por competencias, entra en contradicción con la estructura de actividad curricular semestral del sistema universitario, no sólo por la configuración temporal sino también por los requerimientos que cruzan aspectos de índole administrativa con aspectos de tipo académico. En el ámbito institucional, también se visualizan dificultades referidas a la disposición de recursos económicos para financiar las necesidades que supone la implementación, ya que ésta implicaría no sólo recursos para la docencia, sino también para el acompañamiento, el monitoreo y la supervisión de los aprendizajes de los estudiantes, recursos para contrataciones de académicos especialistas, recursos para materiales, entre otros, lo cual convierte a la propuesta curricular por competencias en un proyecto de alto costo económico.

Finalmente, identificaron como limitación conseguir que la comunidad académica, más allá de quienes conformaron los equipos curriculares, logren entrar en el orden del discurso de las competencias, que adhieran a las nuevas formas de trabajo que los proyectos suponen, y que no lo consideren una amenaza a sus formas tradicionales de desarrollo de la docencia; para ello indicaron la necesidad de desarrollar diversas estrategias para la instalación del modelo, tales como capacitación, inducción y asesorías externas. También señalaron como elementos obstaculizadores de la implementación las características actuales de la docencia impartida, la poca capacidad para el trabajo en equipo y las condiciones para ello, que permitieran realmente la articulación curricular y el desarrollo de procesos de evaluación de competencias.

Como se aprecia, en términos discursivos se produce una difracción del discurso, o sea, una incompatibilidad de los enunciados, ya que si bien es a través de las instancias institucionales como el discurso de las competencias se introduce en el ámbito de la formación inicial docente y encuentra un orden legítimo, las lógicas institucionales frenan las posibilidades de la implementación a la que aspiran sus diseñadores.

\section{CONCLUSIONES}

Se han descrito algunos aspectos de la complejidad de los procesos de inserción del discurso de las competencias en la formación inicial docente. Desde la perspectiva del análisis del discurso de orientación foucaultiana, el propósito de la descripción de las contradicciones no es su superación sino la identificación de asperezas, quiebres y discontinuidades que permiten aproximarnos a la complejidad de las prácticas discursivas.

Nos encontramos con que el discurso de las competencias se inserta en la educación superior universitaria, validando su inclusión mediante enunciados dispuestos en una lógica 
causal, presentando un discurso aparentemente neutro, sólido en sus argumentaciones respecto de su pertinencia, avalado además por una retórica que apela al consenso y la validez. Sin embargo, esta estabilidad de la lógica causal se tensiona con los enunciados en que se identifican serias dificultades y limitaciones para su implementación en los contextos universitarios específicos.

Junto con esto, en esta investigación se pudo observar que la utilización del modelo de competencias no implicó un aporte significativo para una comprensión más compleja del ejercicio docente, sino que más bien se centró en la especificación de los aspectos instruccionales; si bien tal dimensión es altamente relevante, como plantea Tenti Fanfani (2006), es necesaria una integración de la dimensión técnico-instrumental con las dimensiones éticas, sociales y políticas del oficio docente:

La racionalidad técnico instrumental del oficio debe ser fortalecida para potenciar las capacidades del docente en la solución de problemas complejos e inéditos de la enseñanza y el aprendizaje. Pero es preciso acompañar esta dimensión racional técnica del oficio con elementos de tipo afectivo, asociados a la vieja idea de la vocación. Como se dijo arriba, la docencia requiere de un compromiso ético-moral que involucre el respeto, cuidado e interés por el otro, es decir, por el aprendiz concebido como sujeto de derechos. Por último, la docencia no es una actividad neutra, no es un trabajo individual sino doblemente colectivo. Es colectivo en la medida en que el maestro no trabaja solo, sino que la enseñanzaaprendizaje es el resultado de un trabajo en equipo (el docente como intelectual colectivo). Y es colectivo en cuanto trasciende la mera formación de "recursos humanos". En este sentido es una actividad profundamente política, es decir, comprometida con la formación de la ciudadanía activa y la construcción de una sociedad más justa, más libre y por lo tanto, más "humana" (2006:141).

En este contexto, se evidencia la necesidad de que las instituciones expliciten y consoliden las opciones curriculares de sus proyectos formativos, para así entablar diálogos más potentes con las demandas que emanan de las políticas educativas, las cuales se proyectan con decisión a influir cada vez más en los currículos de la formación inicial docente. De lo contrario, la posición de las instituciones se transforma en una alternancia de propuestas responsivas a las contingencias de la política, que no aportan al desarrollo de éstas, y afectan la coherencia y consistencia de sus propios proyectos formativos.

Las instituciones y sus equipos académicos debiesen concretar y explicitar en sus propuestas curriculares el tipo de docente que desean formar, para qué escuela y para qué sociedad. Velar por la articulación de sus propuestas significa identificar los alcances y límites de la formación inicial, evaluar lo que eventualmente podría ser "estandarizable" y lo que no. Esto último con el propósito de disponer de elementos teóricos y prácticos para dialogar críticamente con los discursos que norman la construcción curricular de la formación inicial docente.

El discurso de las competencias en el currículo de la formación inicial puede ser una oportunidad para que las instituciones expliciten con mayor precisión sus propuestas formativas y articulen sus planes de estudio para cumplir con los propósitos declarados, considerando las necesidades del contexto; pero lo que no debiese suceder es asumir prácticas curriculares, sin sentido crítico, no cuestionando su pretensión de verdad, neutralidad o desideologización, sobrevalorando la dimensión técnico instruccional que 
aportan, y pretendiendo que sólo en la definición de competencias o estándares se juega el mejoramiento de la calidad de la formación docente.

Por tanto, la reflexión que se desea relevar es que las propuestas curriculares de formación inicial docente se sustenten en una comprensión más compleja y multidimensional de la realidad educativa, que considere las cercanías y distancias entre el discurso formativo de las instituciones, el de la política y el de los contextos educativos específicos. Finalmente, cabe precisar que dado que la formación inicial docente, al estar inserta en el contexto de la formación superior universitaria, debe pensarse y analizarse desde la complejidad de ésta en el contexto de la globalización neoliberal.

\section{REFERENCIAS BIBLIOGRÁFICAS}

Bonal, X., Tarabini-Castellani, A. y Verger, A. (2007). La educación en los tiempos de globalización: Nuevas preguntas para las ciencias de la educación. En X. Bonal, A. Tarabini-Castellani y A. Verger (Comps.), Educación y globalización. Textos fundamentales (pp. 13-35). Buenos Aires: Miño y Dávila.

Cherryholmes, C. (1999). Poder y crítica: Investigaciones postestructurales en educación. Barcelona: Ediciones Pomares-Corredor.

Dale, R. (2007). Los efectos de la globalización en la política nacional: Un análisis de los mecanismos. En X. Bonal, A. Tarabini-Castellani y A. Verger (Comps.), Educación y globalización. Textos fundamentales (pp. 87-114). Buenos Aires: Miño y Dávila.

Foucault, M. ([1970] 2005). El orden del discurso. (3 ${ }^{\mathrm{a}}$ Ed.). Barcelona: Tusquets. ([1969] 2002). La arqueología del saber (2 ${ }^{\mathrm{a}}$ Reimp.). Buenos Aires: Siglo XXI.

Green, A. (2007). Educación, globalización y el papel de la investigación comparada. En X. Bonal, A. Tarabini-Castellani y A. Verger (Comps.), Educación y globalización. Textos fundamentales (pp. 61-86). Buenos Aires: Miño y Dávila.

Grundy, S. (1994). Producto o praxis del currículum. Madrid: Morata.

Gvirtz, S. y Palamidessi, M. (2005). El ABC de la tarea docente: Currículum y enseñanza. Buenos Aires: Aique.

Ibarra, C. E. (2003). Capitalismo académico y globalización: La universidad reinventada. Educ. Soc., vol. 24, n. 84, 1059-1067. Recuperado de http://www.cielo.br/pdf/es/v24n84/a17v2484.pdf, consultado en marzo de 2011.

Jäger, S. \& Maier, F. (2009). Theoretical and Methodological Aspects of Foucauldian Critical Discourse Analysis and Dispositive Analysis. In R. Wodak \& M. Meyer (Eds.), Methods of Critical Discourse Analysis (pp. 34-61). Los Angeles: Sage.

(2008). Entre las culturas: Caminos fronterizos en el análisis de discurso. Discurso \& Sociedad, vol. 2, n. 3, 503-532. Recuperado de http://www.dissoc.org/ediciones/v02n03/DS2(3) Jaeger.pdf, consultado en junio de 2009.

(2003). Discurso y conocimiento: Aspectos teóricos y metodológicos de la crítica del discurso y del análisis de dispositivos. En R. Wodak y M. Meyer (Comps.), Métodos de Análisis Crítico del Discurso (pp. 61-99). Barcelona: Gedisa.

López Segrera, F. (2007). Notas para un estudio comparado de la educación superior a nivel mundial. En F. López Segrera (Ed.), Escenarios mundiales de la educación superior. Análisis global y estudios de casos (pp. 21-80). Buenos Aires: CLACSO.

(2003). El impacto de la globalización y las políticas educativas en los sistemas de educación superior en América Latina y el Caribe. En M. Mollis (Comp.), Las universidades en América Latina ¿Reformadas o alteradas? (pp. 39-58). Buenos Aires: CLACSO.

Moreno, J. M. (2008). La dinámica del diseño y del desarrollo del currículo: Escenarios para 
la evolución del currículo. En A. Benavot y C. Braslavsky (Eds.), El conocimiento escolar en una perspectiva histórica y comparativa (pp. 311-333). Buenos Aires: Gránica.

Pinar, W., Reynolds, W., Slattery, P. \& Taubman, P. (2008). Understanding Curriculum: An Introduction of Study of Historical and Contemporary Curriculum Discourses. New York: Peter Lang.

Popkewitz, T. y Brennan, M. (Comps.) (2000). El desafío de Foucault. Discursos, conocimiento y poder en la educación. Barcelona: Ediciones Pomares-Corredor.

Rifá Valls, M. (2003). Michel Foucault y el giro postestructuralista crítico feminista en la investigación educativa. Revista Educación y Pedagogía, vol. XV, n. 37, 71-83. Recuperado de http:// aprendeenlinea.udea.edu.co/revistas/index.php/revistaeyp/article/viewFile/5978/5387, consultado en marzo de 2012.

Santos, B. (2005). La universidad en el siglo XXI. Para una reforma democrática y emancipadora de la universidad. México D.F: Centro de Investigaciones Interdisciplinarias en Ciencias y Humanidades, Universidad Nacional Autónoma de México.

Schiro, S. (2008). Curriculum Theory: Conflicting Visions and Enduring Concerns. Los Angeles: Sage.

Silva, T. T. (2001a). Espacios de identidad: Nuevas visiones sobre el currículum. Barcelona: Octaedro.

. (2001b). Dr. Nietzsche, curriculista con aportes del profesor Deleuze: Una mirada post estructuralista de la teoría del currículum. Revista Pensamiento Educativo, vol. 29, n. 2, 15-36. . (1998). Cultura y currículum como prácticas de significación. Revista de Estudios del Currículum, vol. 1, n. 1, 59-76.

Slaughter, S. \& Rhoades, G. (2004). Academic Capitalism and the New Economy. Markets, State, and Higher Education. Baltimore and London: The Johns Hopkins University Press.

Tenti Fanfani, E. (Coomp.). (2006). El oficio docente. Vocación, trabajo y profesión en el siglo XXI. Buenos Aires: Siglo XXI. 
